\title{
Damping As Structural Integrity Index
}

\section{Sofia D. Panteliou*}

Department of Mechanical Engineering and Aeronautics, University of Patras, Greece

Damping plays an important role in technical practice. Coulomb (1784) (Memoir on Torsion) describes experiments where damping in torsional vibrations is due to material defects, while according to Lazan [1] it is important for many load types.

Degradation of structural integrity of materials is related to strength decrease. Evaluation of the effect of defects on materials strength, especially in fatigue and brittle fracture, is very important consideration in engineering technology. Pre-existing pores, cracks and flaws, in addition to those produced during service due to fatigue or static loading, influence the behavior of a material towards mechanical stresses.

The presence of a defect (i.e. pore, crack) in a structural member according to Dimarogonas [2] introduces a local flexibility affecting its vibration response and depending on the vibration amplitude, thus leading to a coupled system, with new harmonics in the spectrum.

Damping is also a very important material property when dealing with vibrating structures from the point of view of vibration isolation in many applications: bearings, filters, aircraft parts, and generally structures made out of porous materials or facing crack development. For each material there are many mechanisms [1] generating damping.

When a material is subjected to a stress field, there are always temperature fluctuations throughout its volume due to thermo elastic effect. The resulting irreversible heat conduction leads to entropy production which in turn is the cause of thermodynamic damping. The latter is attributed to irreversible temperature effects within the material, is linearly dependent on absolute temperature and independent of stress level, thus being an important alternative in structural integrity assessment through dynamic evaluation of defective structures.

Analytical calculation of thermodynamic damping was first studied by Zener [3]. From the 60s several publications on the use of damping appeared in which, Mechanics and Mechanisms of Material Damping were revealed and damping was identified as a material and system property. In the 90s, however, a leap in the field has been accomplished, when the use of thermodynamic damping was elaborated and lead to improved tools for structural integrity assessment and damping measurement techniques, including applications on a variety of materials (i.e. conventional materials, metal-matrix composites, fiberreinforced metal-matrix composites, etc.).

The use of the thermodynamic theory of damping to find material and system damping due to material porosity in a homogeneous, isotropic, elastic bar with uniformly distributed ellipsoidal cavities under alternating uniform axial stress was introduced by Panteliou and Dimarogonas [4]. It was shown that thermodynamic damping accounts only for the porosity and can be used as a measure of change in material porosity.

A crack in a structure is known to lead to changes in damping because a plastic zone appears along the crack edge. An analytical relationship between crack depth and damping was presented by Panteliou et al. [5], where use of damping as indicator of crack severity was made.

Up to now the following have been achieved:
1. Development of a rigorous thermodynamic damping theory.

2. Analytical tool for calculation of thermodynamic damping.

3. Further work on the effect of porosity and cracks on damping.

4. The effect of the propagating crack, if the time of propagation is not greatly larger than the period of vibration.

5. Analytical formulation of damping change due to changes in porosity and crack propagation, as an alternative means of structural integrity identification and monitoring.

6. Design and construction of dedicated devices for damping measurement which have been used for testing.

7. A wealth of experimental investigations on defective structures made out of conventional and advanced materials.

8. Recognition of damping as essential valuable tool for monitoring structural changes.

\section{Conclusion}

The potential of damping to be used as porosity and crack identifier was revealed during the last decade, thus rendering damping a tool for monitoring structural integrity, in a wide range of applications, mechanical and biomedical, in an advantageous manner in comparison to all existing conventional techniques.

On the other hand, measurement of damping at one of the modes of vibration results in a weighted average of material damping, the weight function being the corresponding natural vibration mode. Different modes can give information for the concentration of defects (i.e. porosity) at different places throughout the structure. This is a topic of interest still unanswered for further research. Additionally, a consistent study of thermal effects due to porosity or cracks in vibrating structures is yet to be performed.

\section{References}

1. Lazan B J (1968) Damping of materials and members in structural mechanics Pergamon Press 317.

2. Dimarogonas AD (1996) Vibration of cracked structures: A state of the art review. Eng Fract Mech 55: 831-857.

3. Zener C (1937) Physical Review 52: 230-235.

4. Panteliou SD, Dimarogonas AD (1997) Thermodynamic damping in porous materials with ellipsoidal cavities. J Sound Vib 201: 555-565.

5. Panteliou SD, Chondros TG, Argyrakis VC, Dimarogonas AD (2001) Damping Factor as an Indicator of Crack Severity. J Sound Vib 241: 235-245.

*Corresponding author: Sofia D. Panteliou, Associate Professor, Department of Mechanical Engineering and Aeronautics, University of Patras, Greece, Tel: +30-2610997206, +30-6932-814102; Fax: +30-2610-997207, E-mail: panteliu@mech.upatras.gr

Received August 03, 2012; Accepted August 06, 2012; Published August 08, 2012

Citation: Panteliou SD (2012) Damping As Structural Integrity Index. J Appl Mech Eng 1:e104. doi:10.4172/2168-9873.1000e104

Copyright: (c) 2012 Panteliou SD. This is an open-access article distributed under the terms of the Creative Commons Attribution License, which permits unrestricted use, distribution, and reproduction in any medium, provided the original author and source are credited. 